\title{
539037
}

\section{Anisotropic Nanomechanics of Boron Nitride Nanotubes: Nanostructured "Skin" Effect}

\author{
Deepak Srivastava* \\ NASA Ames Research Center, CSC. Mail Stop T27-A1. Moffett Field, CA 94035-1000

\begin{abstract}
Madhu Menon**
Department of Physics and Astronomy, University of Kentucky, Lexington, KY 40506-0055

and Center for Computational Sciences, University of Kentucky, Lexington, KY 40506-0045

and
\end{abstract}

KyeongJae Cho

Department of Mechanical Engineering, Stanford University, Stanford, CA 94305-4040

(July 24, 2000)

\begin{abstract}
The stiffness and plasticity of boron nitride nanotubes are investigated using generalized tight-binding molecular dynamics and $a b$-initio total energy methods. Due to boron-nitride $\mathrm{BN}$ bond buckling effects, compressed zigzag $\mathrm{BN}$ nanotubes are found to undergo novel anisotropic strain release followed by anisotropic plastic buckling. The strain is preferentially released towards $\mathrm{N}$ atoms in the rotated $\mathrm{BN}$ bonds. The tubes buckle anisotropically towards only one end when uniaxially compressed from both. A "skin-effect" model of smart nanocomposite materials is proposed which will localize the structural damage towards the 'skin' or surface side of the material.
\end{abstract}

Typeset using REVTEX 
The riscovers of carbon (C) nanotubes by lijima. [1] has set off a tremendons explosion in greneral intresst in these quasi one-dimensional structures. The single-mall nanotutes consist of rolled-up graphene sheets of different diameters and chiralities. Depending on the chiralities, the nanotubes can be either metallic or semiconducting. [2-6] Various applications as components in molecular electronics or nanostructured quantum wire have been proposed and investigated both theoretically and experimentally. Investigations of mechanical and materials properties have also been carried out and reveal these materials to have the highest stiffness and strength characteristics.

The possibility of the existence of nanotubes made of non-carbon elements has also received significant attention. In particular, tubes made of boron nitride $(B N)$ have been the subject of significant investigations, both theoretically and experimentally. [7-16] Successful synthesis of pure boron nitride nanotubes by arc discharge between a BN-packed tungsten anode and a copper cathode was reported initially. [11] Alternative methods for yield in large quantities and reduced number of layers, including single-wall tubes, have also been developed. [12] Moreover, in a recent yet novel method, a mixture of bundles of single-wall C nanotubes and boron trioxide has been thermally treated at 1523-1623K under nitrogen flow to convert single-wall $\mathrm{C}$ nanotube bundles into pure $\mathrm{BN}$ nanotube bundles via substitutional doping. [13]

Theoretical investigations of $\mathrm{BN}$ nanotubes have been restricted mainly to comparing and contrasting the behavior of $\mathrm{BN}$ nanotubes with similar $\mathrm{C}$ nanotubes. The initial studies were primarily focused on the electronic behavior $[7,8]$ with the finding that BN nanotubes are insulating with large band-gaps $(5.5 \mathrm{eV}[8])$. The structures were not relaxed in these $a b$-initio electronic structure investigations. Recent studies using both ab initio density functional and static and dynamic non-orthogonal tight-binding methods have addressed the structural and nanomechanical issues as well. [14-16] Two prominent structural features have been noted in all the three studies. [14-16]

(i) Bond frustration effect: Presence of $\mathrm{B}-\mathrm{B}$ and $\mathrm{N}-\mathrm{N}$ bonds in nanotubes make them structurally unstable. [17] (ii) Bond rotation effect: Structural relaxation causes each BN 
bond to be slightly rotated so that marh $V$ is rotated ont and $B$ is entatert inter the surfare of a BN nanotube. Thus the BN nanotube has a saw-touth or serrated surface with microscopically rotated BN bonds (Fig.1a). An important consequence of the bond frustration effect is that the odd numbered topological ring defects such as pentagons and heptagons are structurally unstable. $[14,16]$ This effect, combined with the experimental observation that BN nanotubes are generally never seen to be closed by fullerene type of caps, [12] has led to predictions on the correlation between the observed cap structures and tube chiralities. [16] Also, the zigzag BN nanotubes have been predicted to be more stable and easily made in experiments. [16] Recent experiments have indeed confirmed that the zigzag arrangement dominates $\mathrm{BN}$ nanotubes. [18] The above findings indicate that there may be significant structural and nanomechanical differences between $\mathrm{C}$ and $\mathrm{BN}$ nanotubes.

In this Letter, we investigate the implications of the $\mathrm{BN}$ bond rotation, and the serated nature of the BN nanotube surface, on the nanomechanics of the nanotubes. The rotated $\mathrm{BN}$ bonds are aligned parallel to the tube axis in zig-zag nanotubes and perpendicular to the tube axis for arm-chair nanotubes. Consequently, the strained BN bond is expected to affect the compression and tension behavior in zig-zag nanotubes and bending and torsion behavior in arm chair nanotubes. In isotropically compressed zigzag BN nanotubes, our quantum molecular dynamics simulations reveal novel anisotropic strain release and plastic buckling, which are attributed directly to the BN bond rotation. Specifically, the nanotubes release strain and plastically deform preferentially along one direction only. This observation leads us to propose a concept of nano-structured "skin effect" in axially aligned zigzag BN nanotube reinforced composites which will tend to transfer external axial strain induced damage towards the outer "skin" or surface side, while leaving the inner "core" side of the material intact.

The theoretical methods used in the present work are the generalized tight binding molecular dynamics (GTBMD) scheme, of Menon and Subbaswamy, [6] which allows for dynamic relaxation of the system with no symmetry constraints, and ab-initio density functional theory (DFT) using the pseudo-potential method $[19,20]$ for energetics comparisons. Successful 


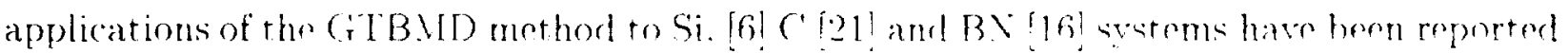
rlsewhere. In the present work we use the GTBMD mothod for hetero-atumic systerms [22] to obtain structural and nanomechanical characterization of BV nanotubes. The relaxation of uncompressed zigzag, arm chair and chiral $\mathrm{BN}$ nanotubes using the scheme produced the BN surface bond rotation effect, [16] in very good agreement with results obtained using static non-orthogonal tight binding [15] and ab initio molecular dynamics methods. [14]

In our study of the effects of BN bond rotation, we consider the axial compression of a relaxed $(8,0)$ BN nanotube. The axial compression is achieved by keeping the edge atoms transparent to the forces generated in the GTBMD method. The positions of the edge atoms are moved axially inward at a fixed rate to compress the nanotube. Each $1 \%$ compression in the linear response regime, and $0.25 \%$ compression near the elastic limit, are accompanied by a GTBMD relaxation of 900 steps. Corrections for edge atom relaxation are similar to that described recently for compression of a $\mathrm{C}$ nanotube, [23] and are incorporated in the stressenergy versus strain plot shown in Fig.2. At the elastic limit $(14.25 \%$ compression of $(8,0) \mathrm{BN}$ nanotube), the compressed tube was allowed to equilibrate for 5000 GTBMD steps, during which a spontaneous "anisotropic" strain release and resulting local plastic deformation were observed. In the following we discuss the Young's modulus and the anisotropic plastic deformation of zigzag BN nanotubes. [24]

The starting configurations of the BN nanotubes had the "perfect" smooth geometry that is typical of carbon nanotubes. The symmetry unconstrained relaxation results in a saw-tooth or "rippled" surface in which the B atoms rotate inward to an approximately planar configuration, whereas the $\mathrm{N}$ atoms move outward into a corresponding pyramidal configuration. For example, the rotation of a $\mathrm{BN}$ bond in comparison with a non-rotated $\mathrm{C}-\mathrm{C}$ bond in a $\mathrm{C}$ nanotube is shown in Fig. 1. The accumulation of strain energy, as the tube is compressed at both ends, as a function of percentage strain is shown in Fig.2. For comparison we also show the recently reported results, using the same method. [23] for a zigzag $(8,0) \mathrm{C}$ nanotube in the same figure. For low applied strain (1-5\%), Young's modulus in the two cases was computed and compared by both GTBMD and ab-initio methods. The 
computed Youmg's modulus of 1.2 TPa for an (8.0) B.V nanotube be GTBMD method is about $92 \%$ of that for a similar C nanotube (1.3 TPa) using the same method. The Young's modulus as computed by the ab-initio method is found to be $1.3 \mathrm{TPa}$. which is about $93 \%$ of the value reported [23] for similar C nanotube (1.4 TPa) using the same ab-initio method. $[19,20]$ This is also in agreement with static non-orthogonal tight-binding results of Hernandez et. al., [15] where it was noted that Young's moduli of a wide variety of BN nanotubes are within $80-90 \%$ of the similar $\mathrm{C}$ nanotubes. In Fig. 2, we also note that the elastic limit of an $(8,0) \mathrm{BN}$ nanotube is larger than that of an $(8,0) \mathrm{C}$ nanotube. This might be expected, since for the similar structure, the lower value of Young's modulus in a BN nanotube is compensated by higher value of the strain to generate forces of similar strength at the elastic limit in the two cases. [25] Additionally, the increase in the elastic limit can also be attributed to the rotated $\mathrm{BN}$ bonds as will be explained below.

The microscopic nature of the anisotropic strain release and the resulting plastic deformation are shown in Fig. 3. At the beginning of the GTBMD relaxation at $14.25 \%$ strain, as shown in Fig. 3a, small deviations from cylindrical cross-section appear near the two ends where strain accumulations are maximum. This is similar to what was reported for a similar compression of $\mathrm{C}$ nanotube as well. [23] The structure starts to undergo a plastic deformation in Fig. 3 (b-e). This plastic deformation towards the right end is driven by the anisotropic release of the accumulated strain energy in the undeformed region of the tube. The strain release occurs preferentially towards $\mathrm{N}$ atoms as leading side of the rotated BN bonds where $\mathrm{N}$ atoms have rotated outward and $\mathrm{B}$ atoms have rotated inward. This process is facilitated by a correlated "sliding" of $\mathrm{N}$ atoms further outward and B atoms further inward. Note that the outward sliding of $\mathrm{N}$ atoms is energetically favorable as it tends to reduce the strain due to the curvature of the tube. The inward sliding of B atoms, however, is hindered as it tends to increase the curvature induced strains. Furthermore, there is also a chemical "repulsion" between neighboring B atoms, preventing the formation of energetically unfavorable B-B bonds. An anisotropy is, therefore, induced in the structure due to the opposing characteristics of the radial displacements of the $\mathrm{B}$ and $\mathrm{N}$ atoms, the details 
of which are analyed next.

The radial position distribution function illustrating the arerage radial displacenents of $B$ and $\mathrm{N}$ atoms as a function of tube compression is shown in Fig. ta. At $0 \%$ compression, due to intrinsic BN bond rotation, two peaks are seen in this function; an inner peak at 3.03 A for average $B$ radius and an outer peak at $3.27 \mathrm{~A}$ for arerage $\cong$ radius. At equilibrium, for $0 \%$ compression and for the average $\mathrm{BN}$ bond length of $1.42 \mathrm{~A}$, this corresponds to an average BN bond rotation of about 9.7 degrees away from being parallel to the tube axis. As the tube is gradually compressed, as shown in Fig. 4a, both the separated B and N peaks move outward and also further apart from each other indicating an increase in the $\mathrm{BN}$ bond rotation angle from about 9.6 degrees at $0 \%$ compression to about 13.2 degrees at $12 \%$ compression. As indicated above, this means that a significant fraction of the axial strain is accommodated by the displacements of $\mathrm{B}$ and $\mathrm{N}$ atoms perpendicular to the tube axis in a correlated manner. The $\mathrm{N}$ atoms are pushed further out while the $\mathrm{B}$ atoms are forced further into the tube.

The relative radial displacements of $\mathrm{B}$ and $\mathrm{N}$ atoms, with respect to their initial radial positions in the uncompressed tube, can be quantified by the relative shifts of $\mathrm{B}$ and $\mathrm{N}$ peaks in Fig. $4 \mathrm{a}$ and are shown in Fig. $4 \mathrm{~b}$ (inset). The relative displacements of both $\mathrm{B}$ and N atoms increase as function of tube compression. However, for all values of compression, the $\mathrm{N}$ atoms are radially displaced more than the $\mathrm{B}$ atoms. The excess strain as the tube is compressed is accommodated more by the larger outward displacements of $\mathrm{N}$ atoms than by the smaller displacements of B atoms. At elastic limit, tube failure initially occurs at both ends (Fig. 3a). The resulting strain release in the center region, however, is anisotropically driven towards $\mathrm{N}$ atoms, as leading sides of the rotated $\mathrm{BN}$ bonds, because it is still energetically favorable to push or "slide" $\mathrm{N}$ atoms further out to accommodate the released strain. The strain release occurs in a concerted outward motion of $\mathrm{N}$ atoms which was observed in the animation of the quantum dynamics of the simulation. At the end, the resulting plastic buckle occurs only towards one end and not the other (Fig. 3b-e).

We analyzed the structure in the buckled region by a bond length distribution func- 
tion analysis and found peaks corresponding to B.N bond loneths of about 1 it in the buckled region. This corresponds to about $5 \%$ compressed BX bond longthin in cubic-BN (experimentally observed BN bond length in uncompressed cubic-BN is about $1.37 . A$. [26]). Similarly to the compression and plastic collapse of a $\mathrm{C}$ nanotube reported recently. [2:3] the plastic deformation in B. is also accommodated by an $s p^{2}$ (three-fold coordination) to $s p^{3}$ (four-fold coordination) type bonding transition at the location of the buckle. The main difference is that $\mathrm{N}$ atoms are driven preferentially outwards as compared to $\mathrm{B}$ atoms. On removing the compression constraints and letting the system relax by the GTB.ID method, the tube was found not to relax back to the initial uncompressed structure. This confirmed the plastic nature of the buckle. As expected, the simulations of armchair nanotube of the same radius did not show any anisotropic strain release mechanism when subjected to the similar axial strains. This is because buckled BN bonds in an armchair nanotube are aligned perpendicular to the tube axis and not parallel.

Based on the anisotropic strain release and the resulting plastic deformation mechanism, a hypothetical composite material reinforced by axially aligned zigzag BN nanotubes can be proposed. The proposed material will exhibit a nanostructured "skin" effect in the sense that the material will have an anisotropic response to external axial strains. When subjected to large external axial strains, the material will react by minimizing the damage to the inner core side, while transferring all the damage to the outer "skin" or surface side. This skin effect will play an important role under external impulse such as in a shock wave, since the composite material will not have enough time to develop a long wavelengh geometric instability such as nanotube buckling under uniaxial compression. Such a hypothetical material, if synthesized, will have useful applications in the transportation, aerospace, defense, and armor industries.

In summary, we have investigated the nanomechanics and plastic deformation of axially compressed BN nanotubes. The BN nanotubes are found to be almost as stiff as similar C nanotubes. Furthermore, for the first time, we have found an anisotropy in the strain release 
and plastic deformation mechanisms in zig7ag B.V nanotubes. The anisotropic strain release is driven by ontward displarements of $\mathrm{N}$ atoms accompanied by inward displacements of $\mathrm{B}$ atoms with larger displacements for $\mathrm{N}$ atoms. This phenomenon and underlying mechanisms as explained by the average relative displacements of $\mathrm{N}$ and $\mathrm{B}$ atoms are general and valid also at smaller values of strain. A hypothetical composite material exhibiting nanostructured "skin" effect, or anisotropic shock absorbing properties is proposed that will have useful applications in a wide variety of industries.

GTBMD relaxation and analysis of the results were performed (DS) at NAS computational facility at NASA Ames Research Center. Part of this research (MM) is supported through grants by NSF (OSR 99-07463, MRSEC Program under award number DMR9809686), DEPSCoR (OSR 99-63231 and OSR 99-63232), NASA Ames Research Center, and the University of Kentucky Center for Computational Sciences. The ab-initio calculations (KJ) were performed on a T90 at SDSC allocated through the NPACI Grant "Nanoscale Materials Simulations." 


\section{REFERENCES}

*e-mail: deepakcunas nasa.gov

** e-mail: super250 ápop.uky.edu

[1] S. Iijima, Nature 354, 56 (1991).

[2] J. W. Mintmire, B. I. Dunlap and C. T. White, Phys. Rev. Lett. 68, 631 (1992).

[3] R. Saito, M. Fujita, G. Dresselhaus and M. S. Dresselhaus Phys. Rev. B46, 1804 (1992).

[4] N. Hamada, S. Sawada and A. Oshiyama, Phys. Rev. Lett. 68, 1579 (1992).

[5] B. I. Dunlap, Phys. Rev. B49, 5643 (1994).

[6] M. Menon, E. Richter and K. R. Subbaswamy, J. Chem. Phys. 104, 5875 (1996).

[7] A. Rubio, J. L. Corkill, and M. L. Cohen, Phys. Rev. B 49, 5081 (1994).

[8] X. Blase, A. Rubio, S. G. Louie, and M. L. Cohen, Europhys. Lett. 28, 335 (1994).

[9] P. Gleize, M. C. Schouler, P. Gadelle, and M. Caillet, J. Mater. Sci. 29, 1575 (1994).

[10] P. Gleize, S. Herreyre, P. Gadelle, M. Mermoux, M. C. Cheynet, and L. Abello, J. Mater. Sci. Lett. 13, 1413 (1994).

[11] N. G. Chopra, R. J. Luyken, K. Cherry, V. H. Crespi, M. L. Cohen, S. G. Louie, and A. Zettl, Science 269, 966 (1995).

[12] A. Loiseau, F. Willaime, N. Demoncy, G. Hug, and H. Pascard, Phys. Rev. Lett. 76, $4737(1996)$.

[13] D. Goldberg, Y. Bando, W. Han, K. Kurashima, and T. Sato, Chem. Phys. Lett., 308 337 (1999).

[14] X. Blase, A. D. Vita, J.-C. Charlier and R. Car, Phys. Rev. Lett. 801666 (1998).

[15] E. Hernandez, C. Goze, P. Bernier, and A. Rubio Phys. Rev. Lett. 80, 4502 (1998). 
[16] M. Nenon and D. Srivastava. Chem. Phrs. Lett.. 307. 407 (1999).

[17] J. R. Browser, D. A. Jelski, and T. F. George, Inorg. Chem. 31. 154 (1992).

[18] D. Goldberg, Y. Bando, K. Kurashima. T. Sato, Solid State Comm. (in press). (2000).

[19] For more details see a review article: M.C. Payne et al., Rev. Mod. Phys. 64, 1045 (1992).

[20] The ab initio simulations were performed with the total energy density functional theory (DFT) method with the local density approximation (LDA). All calculations were done with the optimized pseudopotential for $\mathrm{B}$ and $\mathrm{N}$ atoms with the cutoff energy of $35 \mathrm{Ry}$. To test the cutoff energy convergence bulk cubic BN crystal was simulated, and the calculated lattice constant $(3.63 \AA)$ and bulk modulus $(398 \mathrm{GPa})$ are in good agreement with the experimental values of $3.62 \AA$ and $391 \mathrm{GPa}$. Higer cutoff energy did not change the calcualted vaules. The $(8,0)$ BN nanotubes were simulated using the supercell containing one unit cell of 32 atoms for a sequence of configurations obtained from the GTBMD simulations. 32,000 plane wave basis functions were used with $3 \mathrm{k}$-point sampling for the Brillouin zone along the tube axis.

[21] M. Menon and K.R. Subbaswamy, Phys. Rev. B 55, 9231 (1997).

[22] M. Menon, to be published.

[23] D. Srivastava, M. Menon and K. Cho, Phys. Rev. Lett., 83, 2973 (1999).

[24] D. Goldberg, W. Han, Y. Bando, L. Bourgeois, K. Kurashima, and T. Sato, J. Appl. Phys., 862364 (1999).

[25] B. Yakobson, private communication (2000).

[26] W. A. Harrison, Electronic Structure and the Properties of Solids (Freeman, San Francisco, 1980). 


\section{FIGIRES}

F[G. 1. (a) A rotated B.V bond at 9.7 degrees away from the tube axis in an (8.0) B.V nanotube in comparison with (b) a nun-rotated $\mathrm{C}-\mathrm{C}$ bond in a similar $\mathrm{C}$ nanotube.

FIG. 2. (a)Strain energy as a function of axial compression in $(8,0) \mathrm{BN}$ (solid) and $\mathrm{C}$ (dotted) nanotubes. Both the curves are computed with the quantum GTBMD method. Inset (b) shows the strain energy minimization for $\mathrm{BN}$ (solid) and $\mathrm{C}$ (dotted) nanotubes at $14.25 \%$ and $12 \%$ strain respectively.

FIG. 3. Five stages of spontaneous plastic collapse of the $14.25 \%$ compressed $(8,0) \mathrm{BN}$ nanotube showing; (a) nucleation of deformations near the two ends, (b), (c) and (d) anisotropic strain release in the central compressed section and plastic buckling near the right end of the tube, and (e) shows the final anisotropically buckled structure where all the plastic damage has been transferred towards the right end of the tube; the left end has completely relaxed with no plastic buckling. The cross-section of the tube in each of the five cases is also shown.

FIG. 4. (a) Radial displacement distribution functions (at different $\%$ strain) of $B$ and $N$ atoms as functions of radial distance from the tube axis. (b) Same as (a) except average relative radial displacements (in \%) of $\mathrm{B}$ and $\mathrm{N}$ atoms with respect to their average initial positions at $(0 \%)$ compression. 


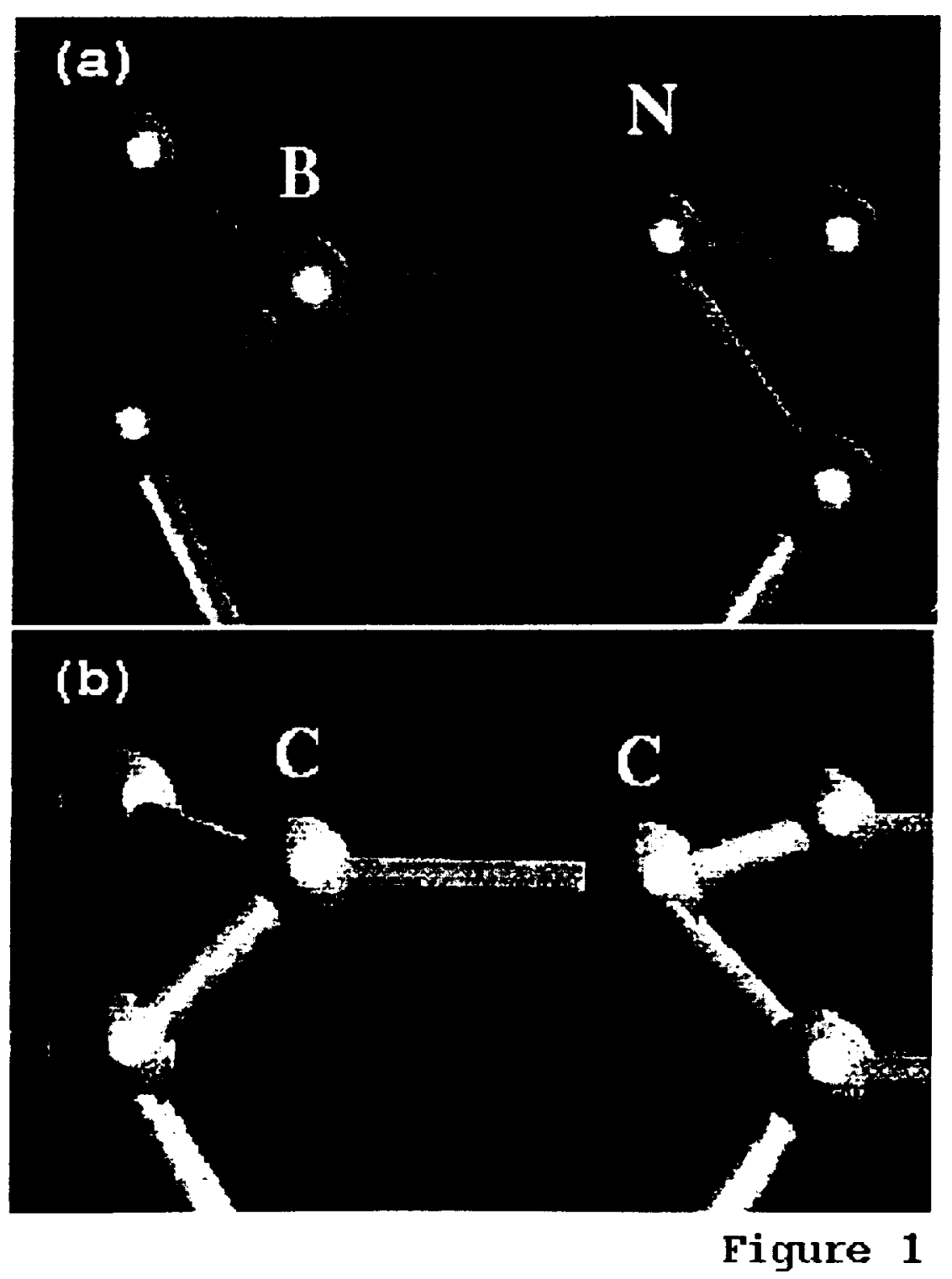




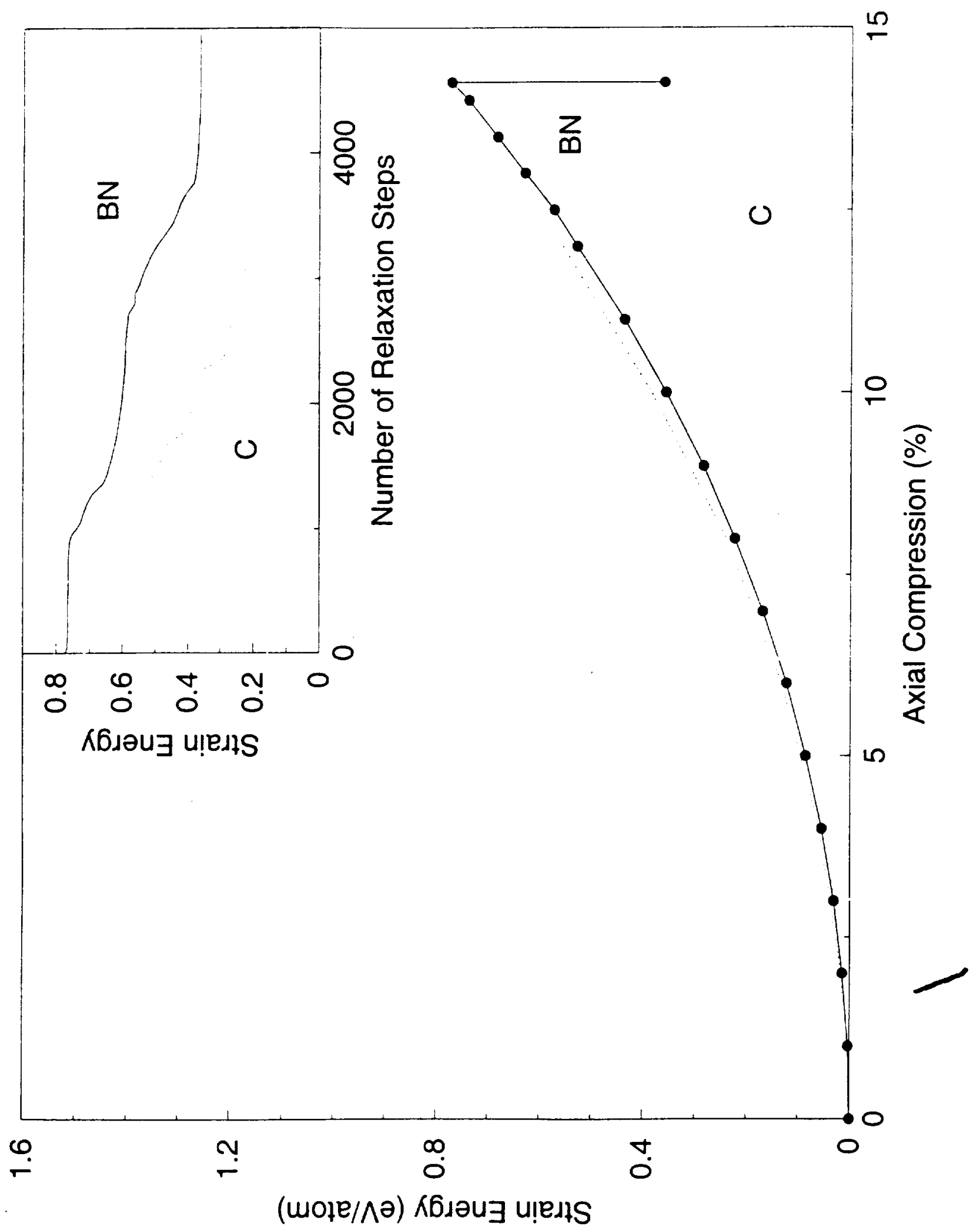



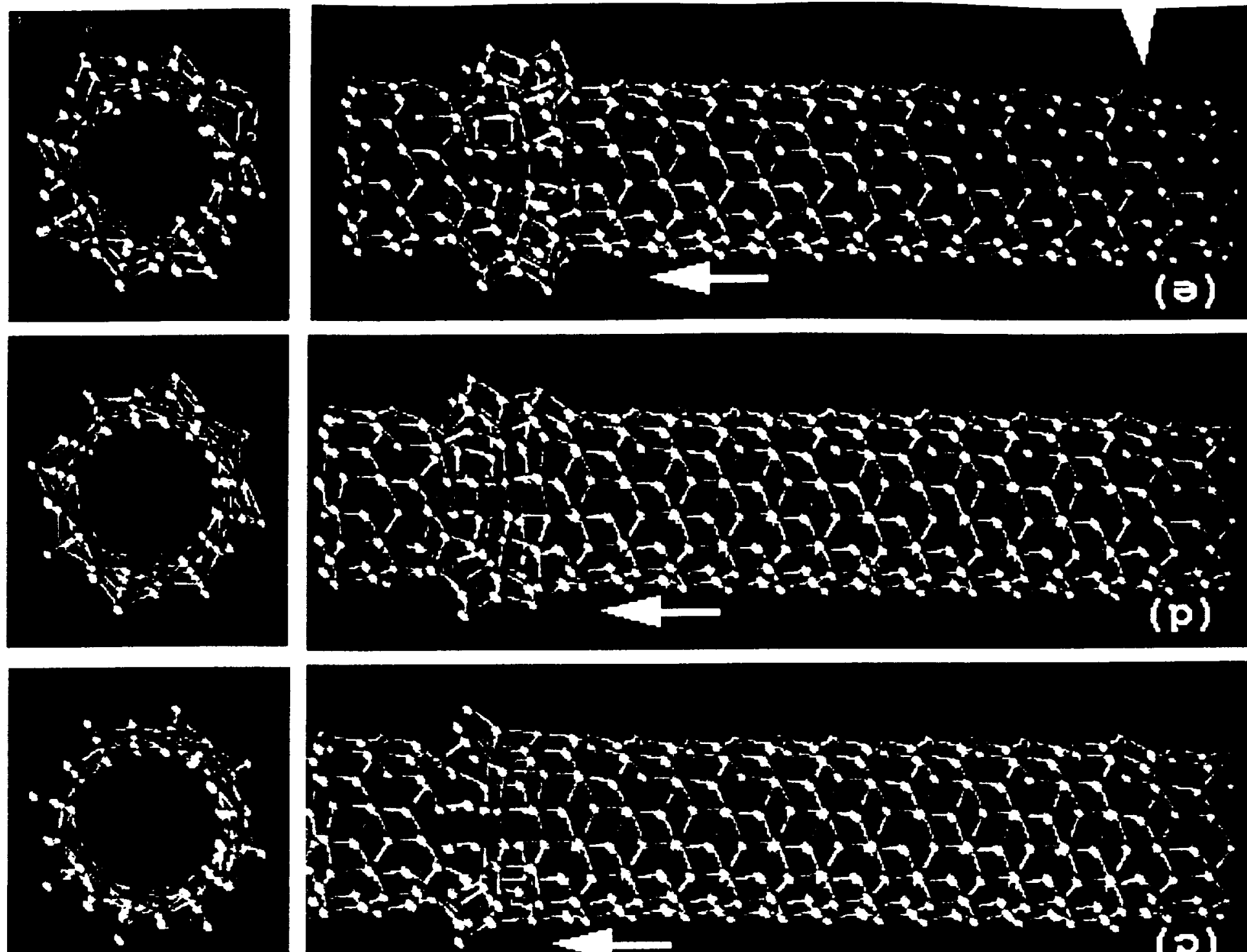

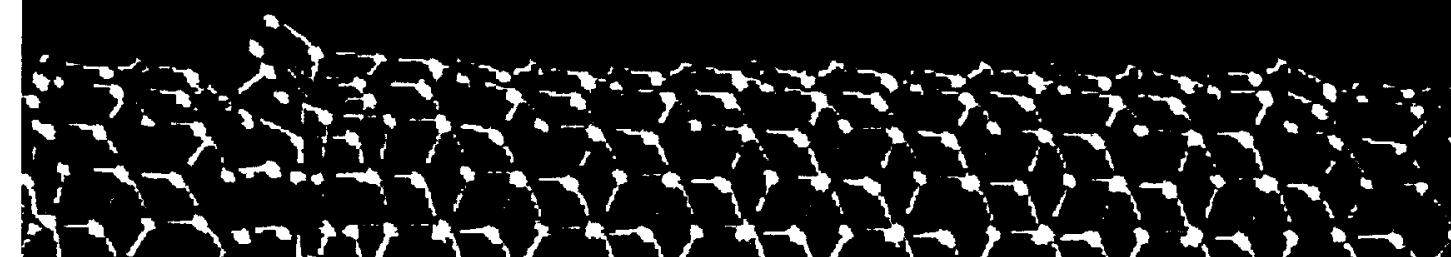
(1)

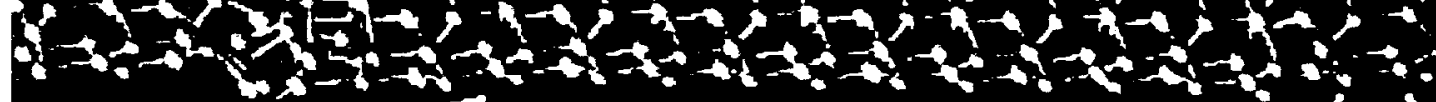
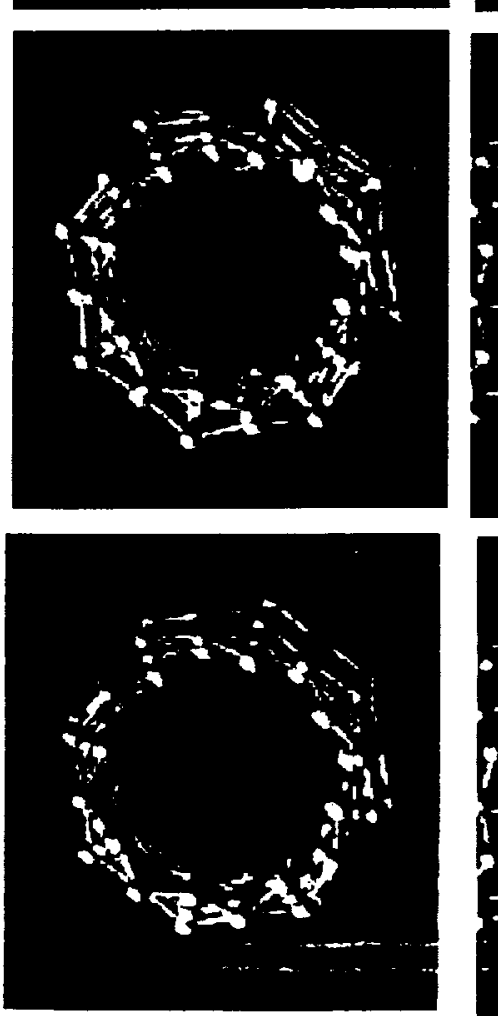

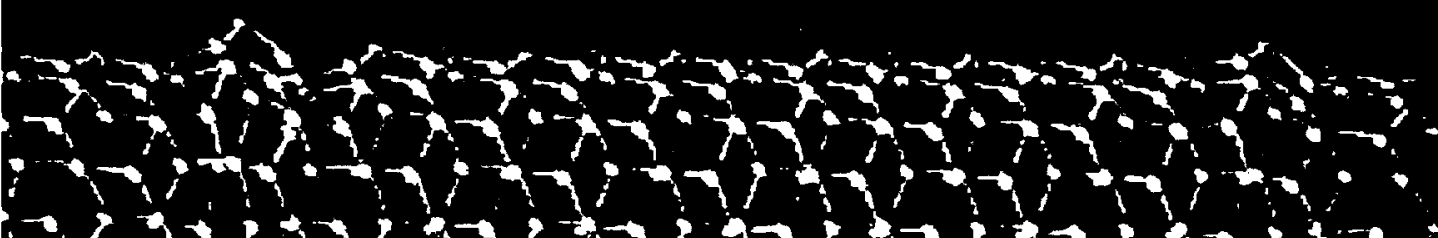

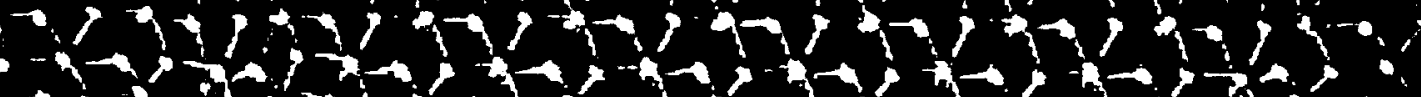

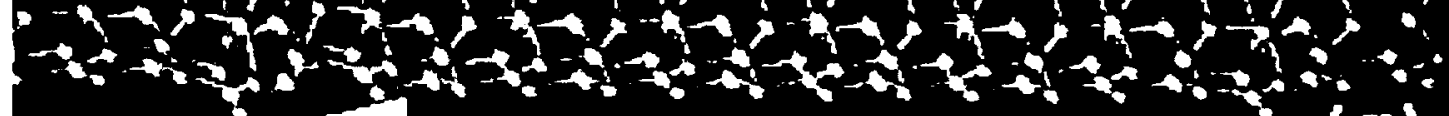




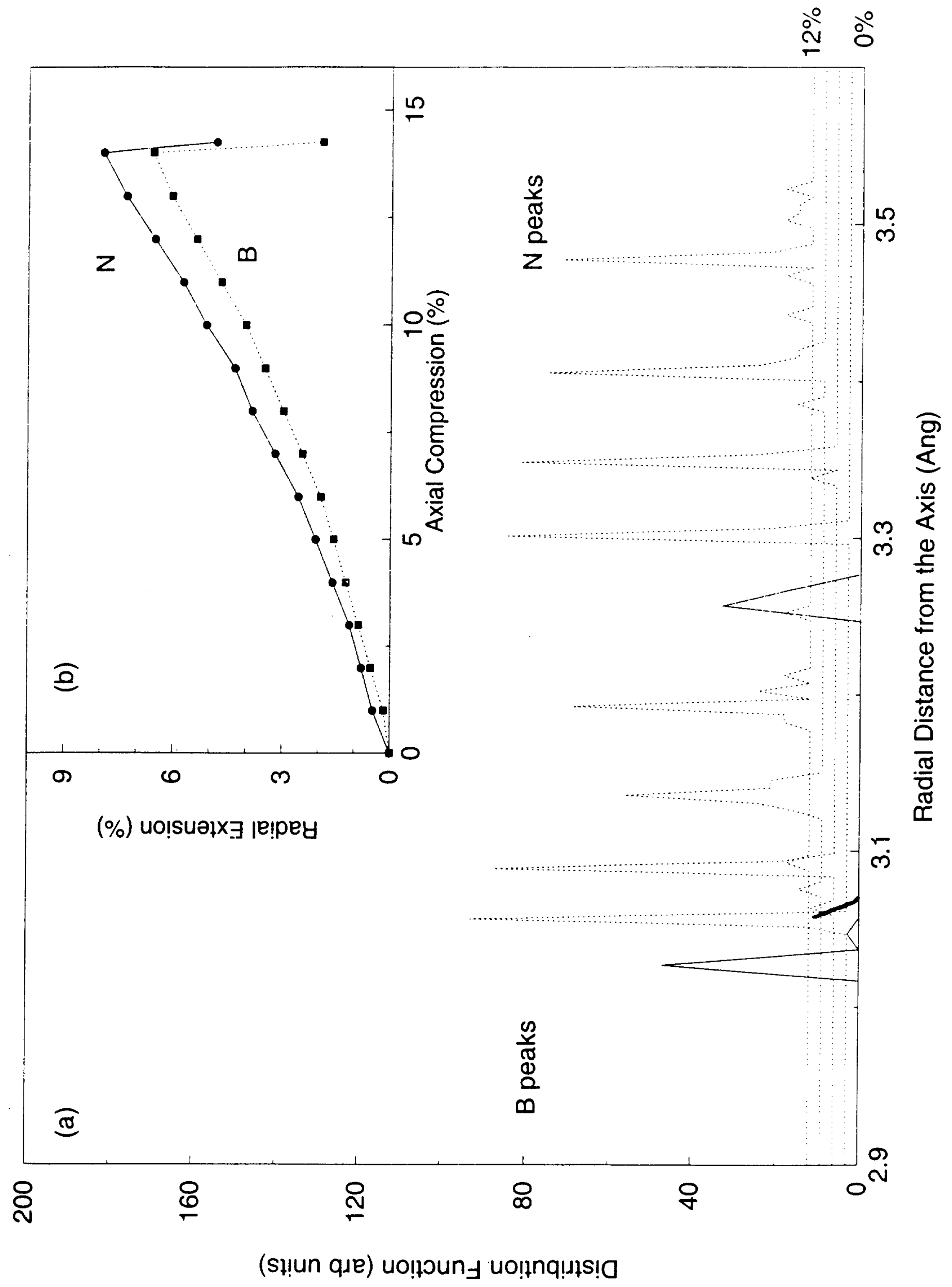

